\title{
Manifestation of Conformal Symmetry in the Light Flavor Baryon Sector
}

\author{
M. Kirchbach and C. B. Compean \\ Instituto de Física, \\ Universidad Autónoma de San Luis Potosí, \\ Av. Manuel Nava 6, San Luis Potosí, S.L.P. 78290, México
}

\begin{abstract}
On the $\mathrm{AdS}_{5}$ cone, conformally compactified to $\mathbf{R}^{1} \otimes S^{3}$, a quark-diquark model of light flavor baryons is developed. The system on this manifold is described in terms of a scalar conformal equation, gauged by the field of a D3 brane whose transversal dimensions have been conformally wrapped over $\mathbf{R}^{1} \otimes S^{3}$. Such a system does not result exactly conformally invariant because the gauge potential slightly modifies the conformal centrifugal barrier of the free geodesic motion. Thanks to this, the model describes the correct mass ordering in the $P_{11}-S_{11}$ pairs through the $N$ spectrum as a combined effect of conformal symmetry breaking, on the one side, and a parity change of the diquark from a scalar at low masses, to a pseudoscalar at higher masses, on the other. We calculate the number of resonances with masses below $2500 \mathrm{MeV}$ needed for the completeness of the above scheme and find a total of 32 "missing" nucleon and $\Delta$ states. Their absence or presence in the respective spectra relates to the degree to which conformal symmetry is realized in the lightest flavor sector. The scheme also allows for a prediction of the dressing function of an effective instantaneous gluon propagator from the Fourier transform of the gauge potential. We find a dressing function that is finite in the infrared and tends to zero at infinity.
\end{abstract}

\section{Introduction}

Applications of brane theory to hadron physics have acquired considerable attention in recent times. The possibility for such applications arose in effect of the intriguing observation [1]-[3] that the asymptotic horizon geometry of the Dirichlet three-brane (D3) of the IIB superstring in ten dimensions considered on the $A d S_{5} \otimes S^{5}$ background, admits a superalgebra that is identical to the super-conformal algebra of the corresponding 4d world-volume field theory when gravity is decoupled. The D3 brane theory has $S U(2,2 / 4)$ as underlying superconformal symmetry whose bosonic isometry $S U(2,2) \otimes S U(4)$, is locally isomorphic to $S O(2,4) \times S O(6)$ [4]. This group happens to coincide with the isometry group of the corresponding asymptotic horizon background, $A d S_{5} \times S^{5}$, a mathematical coincidence that was suggestive of a duality between supergravity around the horizon background, on the one side, and superconformal brane dynamics, on the other. It has been conjectured by Maldacena [5] that zero temperature super Yang-Mills theory residing in the conformal $A d S_{5}$ boundary is likely to capture some of the essential features of high-temperature 3d QCD. Within this context, testing AdS/CFT reduces to the calculation of observables within that very framework and their comparison to the corresponding Lattice results in $3 \mathrm{~d}$ QCD. The duality between D3-brane bulk supergravity and super Yang-Mills open string theories on the conformal boundary of $A d S_{5}$ space-time implies that each CFT state (among them the QCD states in the light flavor sector) can be put in correspondence to a state in the supergravity approximation to string theory on $A d S_{5} \otimes S^{5}$ meaning that spectra in both theories should come out same. As long as the isometry group of the conformal $A d S_{5}$ boundary is the conformal group $S O(2,4)$, the requirement is that the quantum states in the theory on that boundary should populate $S O(2,4)$ unitary representations [6]. This is an essential restriction which strongly limits the number of theories respecting $\mathrm{AdS}_{5} / \mathrm{CFT}_{4}$ and is suggestive of the construction of quark models that respect global conformal invariance. Conformal symmetry is independently to a good approximation a global symmetry of the QCD Lagrangian in the light-flavor sector, a reason for which one can expect spectroscopic data on the light flavor baryons, the nucleon and the $\Delta$, to be especially appropriate in examining the AdS/CFT concept. Implementations of AdS/CFT to hadron physics have been pioneered in refs. [7] within the framework of light-front QCD. There, the conformally invariant two-dimensional (2d) Hamiltonian, $\nu(\nu+1) / \zeta^{2}+\omega^{2} \zeta^{2}$, with $\zeta$ standing for the so called weighted 
impact variable, and $\nu$ related to twist, has been successfully employed in the description of both the meson and light baryon sectors. Further interesting applications of the holographic concept to hadron physics can be found, among others, in refs. [8]- [10]. We here instead implement conformal symmetry into a quark Hamiltonian in three-dimensions which explicitly contains the ordinary angular momentum operator, a circumstance that favorably facilitates identification of predictions to data. We reveal possibility of designing such a model in making use of the conformal map of ordinary Minkowski space, $\mathcal{M}=\mathbf{R}^{1+3}$ onto $S^{1} \otimes S^{3}[1]$, or, $\mathbf{R}^{1} \otimes S^{3}$ [3]. According to [3], correlation functions of CFT on $\mathbf{R}^{1+3}$ can be analytically continued to the full Einstein universe, this because $\mathbf{R}^{1+3}$ can be conformally mapped on $\mathbf{R}^{1} \otimes S^{3}$. The implication of this important observation is that each CFT state on $\mathbf{R}^{1} \otimes S^{3}$ can be brought into unique correspondence with a state of the brane theory on $A d S_{5} \otimes S^{5}$. Consequences on thermal states have been worked out in ref. [11]. We here examine consequences for the systematics of the $N$ and $\Delta$ spectra.

The contribution is organized as follows. In the next section we highlight the procedure of conformal compactification of Minkowski spacetime along the line of ref. [12] and present the conformal equation which will be applied to a quark-diquark (q-(qq)) model of light baryons in section 3. There, we calculate the nucleon spectrum and the mean square charge radii of some of the excited states. Section 4 is devoted to the design of a dressing function of an (instantaneous) effective gluon propagator as a Fourier transform of the gauge potential on $S^{3}$. The contribution closes with brief conclusions.

\section{Conformal compactification of the $\mathbf{A d S}_{5}$ cone to $S^{1} \otimes S^{3} \sim \mathbf{R}^{1} \otimes S^{3}$}

The description of the confinement phenomenon is one of the major goals in the physics of hadrons. Confinement implies exclusion of scattering states and favoring discrete bound states alone. Putting systems on finite volumes is a standard strategy of spectrum discretization [13]. There is a variety of geometries appropriate for preserving the conformal symmetry by the resulting Hamiltonians, the three dimensional sphere, $S^{3}$, being one of them [3], [12], [13]. It has been shown that geometries containing $S^{3}$, such as the $S^{1} \otimes S^{3}$ manifold, can be approached departing directly from $\mathrm{AdS}_{5}$ [1],[2], [12]. The five dimensional manifold $\mathrm{AdS}_{5}$ is defined as a $\mathbf{R}^{2+4}$ subspace according to

$$
u^{2}+v^{2}-x_{1}^{2}-x_{2}^{2}-x_{3}^{2}-x_{4}^{2}=\rho^{2},
$$

where $\rho$ is a fixed parameter. The boundary at infinity of this space is identified with the $A d S_{5}$ cone

$$
u^{2}+v^{2}-x_{1}^{2}-x_{2}^{2}-x_{3}^{2}-x_{4}^{2}=0 .
$$

Flat four-dimensional (4d) Minkowski space time can then be thought of as the intersection of the null hyperplane $v^{2}-x_{4}^{2}=0$ with the $A d S_{5}$ cone, in which case

$$
v^{2}-x_{4}^{2}=u^{2}-x_{1}^{2}-x_{2}^{2}-x_{3}^{2}=0,
$$

and $u$, and $\vec{x}=\operatorname{column}\left(x_{1}, x_{2}, x_{3}\right)$ in turn assume the róles of time and position vector in $\mathbf{R}^{1+3}$, respectively. A quark model placed on the $\mathrm{AdS}_{5}$ cone patterned after eq. (3) allows for a description in terms of light-front variables, a path taken by refs. [7]. We here instead take the different view on Minkowski space as emerging from an $\mathrm{AdS}_{5}$ cone patterned after

$$
u^{2}+v^{2}=x_{1}^{2}+x_{2}^{2}+x_{3}^{2}+x_{4}^{2}=R^{2}, \quad R \neq 0,
$$

in which case the cone compactifies to $\mathcal{M}^{*(1+3)}=S^{1} \otimes S^{3}$. The $S^{1} \otimes S^{3}$ manifold then describes the particular set of $\mathbf{R}^{2+4}$ null rays associated with eq. (4). As long as the isometry group, $S O(2) \otimes S O(4)$, of $S^{1} \otimes S^{3}$, is a subgroup of the conformal group $S O(2,4)$ of regular $(1+3)$ Minkowski space, $\mathcal{M}$, i.e. $S O(2) \otimes S O(4) \subset S O(2,4)$, the above procedure is referred to as conformal compactification of Minkowski space time. Now one can parametrize the $S^{1} \otimes S^{3}$ manifold by the four angles $\tau, \chi, \theta, \varphi$ in accordance with

$$
\begin{array}{ll}
u+i v=R e^{i \tau} & x_{1}+i x_{2}=R \sin \chi \sin \theta e^{i \varphi}, \quad x_{3}=R \sin \chi \cos \theta, \\
x_{4}^{2}+\mathbf{r}^{2}=R^{2}, & r=|\mathbf{r}|=R \sin \chi,
\end{array}
$$


where $R$ is the $S^{3}$ hyper-radius. According to ref. [12], at the microscopic scale this map takes the flat space Minkowski metric to the metric of Einstein's $\mathbf{R}^{1} \otimes S^{3}$ cylinder,

$$
d s^{2}=\Omega^{-2}\left(-\mathrm{d} \tau^{2}+\mathrm{d} \chi^{2}+\sin ^{2} \chi\left(\mathrm{d} \theta^{2}+\sin ^{2} \theta \mathrm{d} \varphi^{2}\right)\right),
$$

with $\Omega$ being the conformal factor. In this way, $S^{1} \otimes S^{3} \simeq \mathbf{R}^{1} \otimes S^{3}$ isomorphism is established

\subsection{Free geodesic motion on $S^{3}$ and the conformal free rigid rotor.}

Within the metric of eq. (6), and the conformal factor being absorbed by the wave functions, the following conformally invariant massless scalar field equation has been found in ref. [12],

$$
-\hbar^{2} \widehat{\square} \psi+\mu^{2} \psi=0
$$

Here, $\mu^{2}$ is a conformal constant [12], $\widehat{\square}$ stands for the angular part of the 4d Laplace-Beltrami operator, which we here choose to express in terms of $\mathcal{K}^{2}$, and $\mathbf{L}^{2}$, the operators of the squared four- and three dimensional angular momenta as,

$$
=-\frac{1}{R^{2}} \frac{\partial^{2}}{\partial \tau^{2}}-\frac{1}{R^{2}} \mathcal{K}^{2}, \quad \kappa=\frac{1}{R^{2}}, \quad-\mathcal{K}^{2}=\left[\frac{1}{\sin ^{2} \chi} \frac{\partial}{\partial \chi} \sin ^{2} \chi \frac{\partial}{\partial \chi}-\frac{\mathbf{L}^{2}(\theta, \varphi)}{\sin ^{2} \chi}\right]
$$

Furthermore, $\chi \in[0, \pi]$ is the second polar angle on $S^{3}$, while $\kappa$ denotes the curvature. The $\mathcal{K}^{2}$ eigenstates, $\mid K l m>$, are well known to belong to irreducible $S O(4)$ representations of the type $\left(\frac{K}{2}, \frac{K}{2}\right)$, and the quantum numbers, $K, l$, and $m$ define the eigenvalues of the respective four-, three- and two-dimensional angular momentum operators upon the states [14]. The $\mathcal{K}^{2}$ eigenvalues, $\lambda_{K}$, upon $|K l m\rangle$, are known too and given by,

$$
\begin{aligned}
\mathcal{K}^{2}|K l m\rangle=\lambda_{K}|K l m\rangle, & \lambda_{K}=K(K+2), \quad|K l m\rangle \in\left(\frac{K}{2}, \frac{K}{2}\right), \quad K=, 0,1,2, \ldots, \infty, \\
\mathbf{L}^{2}|K l m\rangle=l(l+1)|K l m\rangle, & \left.L_{z}|K l m>=m| K l m\right\rangle, \quad l=0,1, \ldots K, \quad m=-l, . .0, \ldots l .
\end{aligned}
$$

The infinite series of solutions of eq. (9) constitute an $\infty$ d unitary representation of the conformal group that has been built up from the eigenstates of its little group, $S O(4)_{K}$ [4], [15]. This irrep will be frequently termed to as a "conformal band". Therefore, the conformal symmetry aspect of AdS/CFT is adequately captured by the $\mathcal{K}^{2}$ eigenvalue problem, which in this fashion qualifies as a suitable departure point toward the description of conformal excitation modes in two-body systems. Independently, conformal symmetry is also to a good approximation global symmetry of the QCD Lagrangian in the light flavor sector, one more reason why employing the conformally invariant AdS/CFT scenario from above in modeling $N$ and $\Delta$ excitations should be of interest. Upon factorizing the $\tau$ dependence of the solution to eq. (7) as $\exp \left(i \frac{\mathcal{E}}{\hbar \sqrt{\kappa}} \tau\right)$, one arrives at the following angular equation on $S^{3}$,

$$
\left[\hbar^{2} \kappa \mathcal{K}^{2}-\mathcal{E}^{2}+\mu^{2}\right] \mathcal{S}(\chi)=0
$$

Though in angular variables, the conformal eq. (10) has the algebraic form of a Klein-Gordon equation which provides a relativistic description of the free geodesic motion on $S^{3}$ in terms of the eigenvalue problem of the squared $4 \mathrm{~d}$ angular momentum. The spectrum of eq. (10) reads,

$$
\mathcal{E}_{K}^{2}-\mu^{2}=\kappa \hbar^{2} K(K+2)
$$

and represents no more but what one could term to as the spectrum of the "conformal free rigid rotor". Stated differently, one can say that the $\mathcal{K}^{2}$ eigenvalue problem has $S O(4)$ as potential algebra, and the conformal group as dynamical symmetry. 


\subsection{The conformal interacting rigid rotor.}

The next step is introducing interaction on $S^{3}$ in such a manner as to respect the conformal symmetry of the spectrum of the free geodesic motion. For this purpose, use can be made of an observation reported in ref. [17]. There, a broad class of exact string solutions have been constructed by wrapping transversal dimensions of fundamental strings over curved spaces and solving the corresponding curved space Laplace-Beltrami equations for harmonic functions. Specifically on $S^{3}$ it has been shown that a conformal string field, $K(\chi)$, (in the notations of ref. [17]) is obtained from the metric,

$$
d s^{2}=\mathrm{d} u \mathrm{~d} v+K(\chi) \mathrm{d} u^{2}+\mathrm{d} \chi^{2}+\sin ^{2} \chi \mathrm{d} \theta^{2}+\sin ^{2} \theta \mathrm{d} \varphi^{2},
$$

with $K(\chi)$ being a harmonic function on $S^{3}$

$$
\widehat{\square} K(\chi)=0, \quad K(\chi)=\bar{a}+m \cot \chi
$$

This important observation qualifies the cotangent function as a conformal potential on $S^{3}$. A further and independent motivation in favor of employing the cotangent potential in quark models is provided by the observation [16] that the lowest terms in its Taylor expansion coincide with a Coulombic+linear (Cornell) potential. This is best illustrated by the simplistic $\chi=\frac{r}{R} \pi$ parametrization (commonly used in super-symmetric quantum mechanics) for which

$$
-\cot \frac{r}{R} \pi=-\frac{d}{r}+\frac{1}{3} \frac{r}{d}+\frac{r^{3}}{45 d^{3}}+\frac{2 r^{5}}{945 d^{3}}+\ldots, \quad \text { with } \quad d=\frac{R}{\pi}, \quad m>0
$$

holds valid. The Cornell potential [18] has been predicted by lattice QCD simulations [19], on the one side, and has been also independently confirmed within the AdS/CFT context where it emerges as a soft-wall Wilson loop potential [5],[20]. Corrections to the Cornell potential that account for non-perturbative processes, have been calculated within the topological approach [21]. Within the light of this discussion, the finite range of the cotangent potential can be viewed as a particular phenomenological parametrization of the non-perturbative corrections to the Coulombic+linear potential embedded in it. Therefore, the cotangent potential on $S^{3}$, besides being congruent with $\mathrm{AdS}_{5} / \mathrm{CFT}_{4}$, also correctly captures the dynamical aspects of QCD, a twofold advantage that makes it attractive to applications in hadron spectroscopy.

We shall introduce this very potential as a gauge interaction in eq. (8) by means of the replacement,

$$
i \hbar \sqrt{\kappa} \frac{\partial}{\partial \tau} \rightarrow i \hbar \sqrt{\kappa} \frac{\partial}{\partial \tau}+2 G \sqrt{\kappa} \cot \chi-\bar{a}
$$

where we parametrized $m$ in eq. (13) as $m=-2 G \sqrt{\kappa}$. Upon factorizing the $\tau$ dependence of the total wave function as $\exp \left(-i \frac{E}{\hbar \sqrt{\kappa}} \tau\right)$, and after some algebraic manipulations, the interacting Klein-Gordon equation can be cast into the following form,

$$
\begin{aligned}
\left(-\kappa \hbar^{2} \frac{\mathrm{d}^{2}}{\mathrm{~d} \chi^{2}}+U_{l}(\kappa, \chi)\right. & \left.-2 G \sqrt{\kappa}(2 E-2 \bar{a}) \cot \chi-(2 G \sqrt{\kappa})^{2} \csc ^{2} \chi\right) \Psi(\chi) \\
& =\left[(E-\bar{a})^{2}-\mu^{2}-\left(\bar{a}^{2}+(2 G \sqrt{\kappa})^{2}\right)\right] \Psi(\chi) .
\end{aligned}
$$

The second term on the 1.h.s of this equation,

$$
U_{l}(\chi, \kappa)=\hbar^{2} \kappa l(l+1) \csc ^{2} \chi
$$

is the centrifugal barrier of the free geodesic motion on $S^{3}$. Indeed, introducing the new constant,

$$
\alpha(l)=-\frac{1}{2}+\sqrt{\left(l+\frac{1}{2}\right)^{2}-\frac{(2 G)^{2}}{\hbar^{2}}}=l+\Delta l, \quad \Delta l \approx-\frac{1}{2} \frac{(2 G)^{2}}{\hbar^{2}} \frac{1}{l+\frac{1}{2}},
$$


allows to rewrite eq. (16) equivalently to,

$$
\begin{aligned}
{\left[-\kappa \hbar^{2} \frac{\mathrm{d}^{2}}{\mathrm{~d} \chi^{2}}+\mathcal{V}(\chi)\right] \Psi(\chi)=} & \left((E-\bar{a})^{2}-c_{0}\right) \Psi(\chi) \\
\mathcal{V}(\chi)=-2 \beta \cot \chi+\bar{U}_{l}(\chi, \kappa), & \bar{U}_{l}(\chi, \kappa)=\hbar^{2} \kappa \alpha(l)(\alpha(l)+1) \csc ^{2} \chi, \\
\beta=2 G \sqrt{\kappa}(E-\bar{a}), & c_{0}=\mu^{2}-\hbar^{2} \kappa+\bar{a}^{2}+(2 G \sqrt{\kappa})^{2} .
\end{aligned}
$$

The positive sign in front of the square root in eq. (18) ensures that $\bar{U}_{l}(\chi, \kappa)$ approaches the $S^{3}$ centrifugal barrier of the free geodesic motion in the $G \rightarrow 0$ limit, $\bar{U}_{l}(\chi, \kappa) \stackrel{G \rightarrow 0}{\longrightarrow} U_{l}(\chi, \kappa)$. Upon a suitable variable change, differential equations of the type in (19) have been shown [22] to reduce to one of the forms of the hyper-geometric equation whose solutions can be expressed in closed form in terms of the non-classical Romanovski polynomials (here denoted by $\left.R_{n}^{(a, b)}(\cot \chi)\right)$. The resulting explicit formula for the wave functions then emerges as,

$$
\begin{aligned}
\Psi_{K l}(\chi)= & N_{K l} e^{-a \chi}(\sin \chi)^{K+1+\Delta l} R_{n}^{(a, b)}(\cot \chi), \\
a=\frac{2 G(E-\bar{a})}{\sqrt{\kappa} \hbar^{2}(K+1+\Delta l)}, & b=-(K+1+\Delta l), \quad K=n+l,
\end{aligned}
$$

where $N_{K l}$ are normalization constants. Correspondingly, the algebraic equation for the energy takes the form,

$$
(E-\bar{a})^{2}-c_{0}=\hbar^{2} \kappa(K+1+\Delta l)^{2}-\frac{4 G^{2} \kappa(E-\bar{a})^{2}}{\hbar^{2}} \frac{1}{(K+1+\Delta l)^{2}}, \quad l \in[0, K] .
$$

In the interacting case, the principal quantum number, $(K+1+\Delta l)$, in eq. (22) can be at most close to integer, thus relaxing the degeneracy of states within the $(K / 2, K / 2)$ multiplet in eq. (9). Nonetheless, it will still be possible to classify a light baryon spectrum in terms of the $S O(4)$ states $|K l m\rangle$, and this very spectrum will still fall into an $\infty \mathrm{d}$ unitary representation of the conformal group. This because, as we shall see below, the $\Delta l$ contributions will have a detectable effect only on the masses of states with $l=0$ and will be helpful in removing the degeneracy between $P_{2 I, 1}-S_{2 I, 1}$ resonances and reproducing their correct mass orderings, while leaving all the other excitations practically degenerate. In effect, the relativistic framework of the Klein-Gordon equation (16) with a cotangent gauge potential provides the intriguing possibility of having conformal bands whose states are not necessarily perfectly degenerate though they still keep spreading around $S O(4)$ levels. This is quite an interesting situation and contrasts the case of the linear in the energy Schrödinger equation which keeps respecting in the interacting case the degeneracies of the free geodesic motion [23].

\section{Quark-diquark model on $S^{3}$ and the $N$ spectrum}

From now onward we assume dominance of quark-diquark configurations in the internal nucleon and $\Delta$ structures and apply eq. (16) to the description of the relative motion of these two bodies. In considering the diquark as spinless, the total spin, $J$, of the resonance is then obtained through coupling the spin-1/2 of the quark to the $q-(q q)$ relative angular momentum, $l$, according to eq. (9). For the time being, and because of the absence of spin-flavor interactions in the wave equation under consideration, we shall factorize the light flavor quantum number (actually isospin). In due course we shall see that the spectra reported so far do not contradict the above assumptions. As a working hypothesis, to be tested by comparison with data, the nucleon diquark has been set as a scalar for the ground state and the low lying excitation with masses below $1600 \mathrm{MeV}$, and as a pseudo-scalar above. The $\Delta$ diquark has been set as an axial vector in the ground state and as a pseudo-scalar above. The pseudo-scalar diquark is a $P$ wave axial-vector of zero total angular momentum and emerges from coupling the axial spin vector, $\vec{S}=\overrightarrow{1}^{+}$, to an internal vectorial, $1^{-}$, excitation of the diquark according to $\left[\overrightarrow{1}^{+} \otimes \overrightarrow{1}^{-}\right]^{0^{-}}$. The quark-diquark system is described by means of eq. (19). The energies $E$ in eq. (22) will be subsequently re-denoted by $M$ and given interpretation of resonance masses read off from the respective ground state nucleon and $\Delta$ masses. In this 
fashion, the spatial part of the total baryon wave function has been designed to account for conformal symmetry in accord with AdS/CFT on the one side, and with the (approximate) conformal symmetry of the QCD Lagrangian in the light flavor sector, on the other. Next we shall compare the outcome of such a model with data on $N$ and $\Delta$ resonances.

The nucleon spectrum. The spectrum of the nucleon continues being under debate despite the long history of the respective studies (see refs. [24], [25] for recent reviews). Yet, unprejudiced inspection of the data reported by the Particle Data Group [26] reveals systematic and hardly to overlook grouping of the excited states of the baryons of the best coverage, the nucleon $(N)$ and the $\Delta(1232)$. Take as a prominent example the seven $\Delta$ resonances $S_{31}(1900), P_{31}(1910), P_{33}(1920), D_{33}(1940), F_{35}(1905), D_{35}(1930)$, and $F_{37}(1950)$, which are squeezed in the narrow mass band between $1900 \mathrm{MeV}$ to $1950 \mathrm{MeV}$ and which, given the limitations of data accuracies, are practically mass degenerate. This group of resonances consists of 3 parity dyads with spins ranging from $\frac{1}{2}^{ \pm}$to $\frac{5}{2}^{ \pm}$and of a single parity state of maximal spin, $J^{\pi}=\frac{7}{2}^{+}$and its quantum numbers fit into the $K=3$ multiplet in eq. (9) . In the nucleon spectrum, one finds the $N(1440)-N(1535)-N(1520)$ triplet, which would match quantum numbers of $K=1$ in eq. (9). Patterns of similar type do not restrict to these two examples alone but extend to the spectrum of any of the lightest flavor baryons, no matter $N$, or $\Delta$. It verifies directly by inspection that the masssplittings inside the above groups are notably smaller than the splittings between the averaged cluster masses. In this fashion the $\Delta$ spectrum reveals a level structure. The $N$ excitations follow quite same patterns though appear shifted downward in mass by about $200 \mathrm{MeV}$ relative to the $\Delta$ excitations. Attention to this clustering phenomenon in light baryon spectra has been drawn in ref. [27]. In assuming equality of the quantum numbers of the $N$ and $\Delta$ excitations and comparing both spectra, allows to pin down the states missing for the completeness of the scheme. The above clustering phenomenon finds a quantitative explanation in terms of the spectrum of eqs. (19), (22). (9) under the assumption of $q-(q q)^{0^{ \pm}}$as a dominant configuration of internal baryon structure. The parity, $\pi$, of the di-quark, scalar, or, pseudoscalar, is fixed by matching parity, $P_{\max }$, of the highest spin, $J=\left(K+\frac{1}{2}\right)$, of the multiplets in eq. (9), the parity simplex, $J^{P_{\max }}=\left(K+\frac{1}{2}\right)^{P_{\max }}$, as $\pi=(-1)^{K} P_{\max }$.

Missing resonance predictions. The comparison of the spectrum in eq. (22) to data on nucleon resonances [26] is presented in the figure. On the nucleon side the highest spins, $F_{17}$ from the $K=3$, and $H_{31,11}$ from $K=5$ are "missing". The $\mathrm{K}=2$ level is completely "missing". Compared to our previous work [16], the fit places the $\mathrm{N}(1900), \mathrm{N}(1990)$, and $\mathrm{N}(2000)$ resonances at the lower $K=4$ level and leaves instead $P_{11}, F_{15}$, and $F_{17}$ in $\mathrm{K}=5$ unoccupied. The number of "missing" nucleonic states is sixteen. The $\Delta$ spectrum, not presented here, follows similar patterns. We predict a total of 32 resonances "missing" from the $N$ and $\Delta$ conformal bands.

Mass ordering in $P_{2 I, 1}-S_{2 I, 1}$ pairs. The model predicts the correct mass ordering of the $P_{11}-S_{11}$ states through the spectrum. Within the framework of the present study the numerical value of the splitting between such states is entirely due to the gauged centrifugal barrier, $\bar{U}_{l}(\chi, \kappa)$, defined in eqs. (19), which prescribes that $l=1$ states will appear higher in mass than those with $l=0$. The ordering, $P_{2 I, 1}-S_{2 I, 1}$ versus $S_{2 I, 1}-P_{2 I, 1}$ depends on the parity of the diquark. When the diquark is a scalar, spin- $\frac{1}{2}^{+}$and spin- $\frac{1}{2}^{-}$in turn refer to zero and unit underlying angular momenta and are associated with $P_{2 I, 1}$, and $S_{2 I, 1}$ states. In case of a pseudoscalar diquark, the situation reverses. This is the reason for which at the scale of $1500 \mathrm{MeV}$, where the diquark is a scalar, the measured $P_{11}(1440)$ state appears lower in mass than its $S_{11}(1535)$ neighbor. From the $1700 \mathrm{MeV}$ level onward, the parity of the diquark changes to pseudoscalar, and it is $S_{11}$ that is built on top of a zero angular momentum. Consequently, $S_{11}$ states with masses above $1600 \mathrm{MeV}$ appear systematically at lower masses than their nearest $P_{11}$ neighbors. Examples are the $S_{11}(1650)-P_{11}(1710)$, and $S_{11}(2090)-P_{11}(2100)$ pairs. Recall that originally the suggestion of the parity change of the diquark was made with the purpose of matching parity of the highest spins in the fermionic multiplets in eq. (9). Therefore, the reverse mass ordering in the $S_{11}-P_{11}$ pairs above $1600 \mathrm{MeV}$ relative to the $P_{11}(1440)-S_{11}(1535)$ splitting, provides an independent argument in favor of the change of parity of the diquark from scalar to pseudoscalar. 
Electric charge form-factors are the simplest physical observables to calculate, and the corner stone of any spectroscopic model. They reduce to the Fourier transform of the charge density, proportional to $\left|\Psi_{K l}(\chi)\right|^{2}$ in our case. The extraction of the mean square charge radius, $\left\langle\mathbf{r}^{2}\right\rangle$, from the form-factor is then standard and calculated as the slope at origin. We here wish to explore importance of relativistic corrections and choose as illustrative examples the mean square charge radii of the Nucleon, the $P_{11}(1440)$ and $S_{11}(1535)$ resonances. The wave functions entering the calculation are obtained from eq. (21). With the potential parameters fitted to the spectra, we find $\sqrt{\left\langle\mathbf{r}^{2}\right\rangle_{p}}=0.664 \mathrm{fm}$, to be compared to the experimental value of $\sqrt{\left\langle\mathbf{r}^{2}\right\rangle_{p}}=0.8750 \pm 0.008 \mathrm{fm}$ reported by [26]. For the Roper and the $S_{11}(1535)$ resonances, same observable is calculated as $\sqrt{\left.<\mathbf{r}^{2}\right\rangle_{\text {Roper }}}=$ $0.8484 \mathrm{fm}, \quad \sqrt{\left\langle\mathrm{r}^{2}>\mathrm{S}_{11}\right.}=0.8754 \mathrm{fm}$. We obtain the mean square charge radius of the proton-like Roper resonance enhanced by a bit less than $30 \%$ over the proton charge radius. Nonetheless, compared to the proton, the form factor of the $P_{11}(1440)$ takes smaller values. This because our predicted $P_{11}(1440)$ charge density appears slightly arced at origin. An enhancement, though smaller (10\%) has been found by Nagata and Hosaka in ref. [28]. One of the differences between the model by Nagata-Hosaka and the present model is that while in the former both the scalar and axial vector diquarks are weighted by non-zero form factors, we here weight them by the extremal 1 and zero values, respectively. This for the sake of staying as close to conformal symmetry as possible. In the present model, it is the curvature parameter that seems to account for some of the effects governed by the diquark form factors in flat space quark models. Curvature as a phenomenological tool suited in mimicking complicated many-body effects is known to be useful in the description of such complicated many-body problems as Brownian motion, plasma correlations, instanton physics etc. [29], [10]. A reason for which the replacement of the complicated many-body problem of baryon structure (the genuine baryon wave function contains next to (q-(qq) also $3 \mathrm{q}, 3 \mathrm{q}(\bar{q} \mathrm{q})^{n}, 3 \mathrm{q}\left(\mathrm{g}^{n}\right)$ etc. configurations) by the simple q-qq problem on $S^{3}$ turns out to be a useful approximation to reality is that curvature, in combination with the conformal gauge potential, account reasonably for the omitted many body effects.

\section{Dressing function for the gluon propagator}

The gluon propagator, $G_{\mu \nu}^{a b}\left(q^{2}\right)$, in the Landau gauge is defined as

$$
G_{\mu \nu}^{a b}=-i\left[\left(g_{\mu \nu}-\frac{q_{\mu} q_{\nu}}{q^{2}}\right) \frac{G\left(q^{2}\right)}{q^{2}}\right] \delta^{a b}, \quad G\left(q^{2}\right)=\left(1+i \frac{m(q)}{q^{2}}\right)^{-1} .
$$

In this gauge it is known to be transverse in the Lorenz indices, and $m(q)$ stands for the gluon self energy. The function $G\left(q^{2}\right)$ is referred to as the gluon dressing function and can be obtained from solving Schwinger-Dyson equations. In so doing, an especially simple expression for $G\left(q^{2}\right)$ has been reported in [30] as $G\left(q^{2}\right) \sim q^{2}$, meaning finiteness of the gluon propagator in the infrared. The propagator approaches zero in the ultraviolet. More complicated expressions have been calculated in [31]. Independently, a finite gluon propagator in the infrared has also been calculated recently in lattice QCD [32]. In view of these properties of the gluon propagator, it is of interest to calculate the gluon dressing function from the $\mathbf{R}^{1} \otimes S^{3}$ quark model. To do so we apply the Born approximation to $E-V=\sqrt{\mathbf{p}^{2}+\mu^{2}}$ and calculate the instantaneous $\left(q_{0}=0\right)$ gluon propagator in parallel to our recent work [23] as a Fourier transform of the $\cot \chi(r)$ potential on $S^{3}$ employing the integration volume in eq. (5). In Cartesian coordinates the $\cot \chi(r)$ term equals $\frac{x_{4}}{\mid \mathbf{r}}$, and stands in fact for two potentials distinct by a sign and describing interactions on the respective Northern, and Southern hemi-spheres. Correspondingly, their respective Fourier transforms to momentum space become

$$
4 \pi \Pi(|\mathbf{q}|)=\frac{(4 \mu G \sqrt{\kappa})}{\hbar^{2}} \int_{0}^{\infty} d|x||x|^{3} \delta(|x|-R) \int_{0}^{2 \pi} d \varphi \int_{0}^{\pi} d \theta \sin \theta \int_{0 / \frac{\pi}{2}}^{\frac{\pi}{2} / \pi} d \chi \sin ^{2} \chi e^{i|\mathbf{q}| \frac{\sin \chi}{\sqrt{\kappa}} \mid \cos \theta} \cot \chi
$$

where the $\delta(|x|-R)$ function restricts $\mathbf{R}^{4}$ to $S^{3}$. It is the requirement on invertability of this transformation, addressed here for the first time, that demands for distinguishing between momentum space potentials on the 


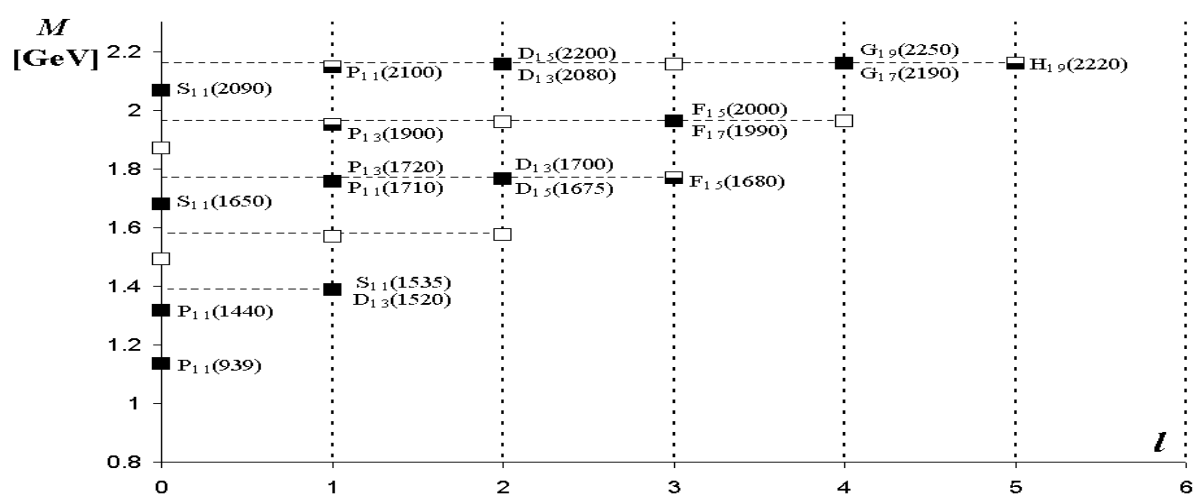

Figure 1: Assignments on the $l / M$ grid of the reported [26] $N$ excitations to the $\mathbf{R}^{1} \otimes S^{3}$ spectrum in eq. (22). The distribution of the reported (exp) resonances over the predicted (th) states has been obtained from running a four parameter least mean square data fit, i.e. minimizing $\sigma=\sqrt{\frac{1}{N} \Sigma_{i=1}^{i=N}\left(M_{\mathrm{th}}^{(i)}-M_{\mathrm{exp}}^{(i)}\right)^{2}}$. The sum includes all the reported resonances. The best fit that also predicts a reasonable value for the proton mean square electric charge radius, is $\sigma_{\min }=0.0855 \mathrm{GeV}$ has been obtained for the following potential parameters: $G=0.0493 \mathrm{GeV} \cdot \mathrm{fm}$, $R=0.9814 \mathrm{fm}$, and $\mu=0.3213 \mathrm{GeV}$, and $\bar{a}=0.932 \mathrm{GeV}$.. The excitations, $E$, set to masses, have been read off from the nucleon mass. Full and empty squares mark measured and "missing" resonances, respectively.

Northern and Southern hemispheres of $S^{3}$. The first potential goes with $\chi \in\left[0, \frac{\pi}{2}\right]$, corresponds to a positive $x_{4}$, and describes an increasing $|\mathbf{r}| \in[0, R]$. The second one refers to $\chi \in\left[\frac{\pi}{2}, \pi\right]$, a negative $x_{4}$, and describes a decreasing $|\mathbf{r}| \in[R, 0]$. To prove the invertability of the integral transform, it is instructive to cast the transformation integral in eq. (24) to the following equivalent form,

$$
4 \pi \frac{\Pi(|\mathbf{q}|)}{\frac{(4 \mu G \sqrt{\kappa})}{\hbar^{2}}}= \pm \int_{0}^{R} \mathrm{~d} r \frac{r^{2}}{\sqrt{R^{2}-r^{2}}} \frac{\sqrt{R^{2}-r^{2}}}{r} \int_{0}^{2 \pi} d \varphi \int_{0}^{\pi} d \theta \sin \theta e^{i \vec{q} \cdot \vec{r}}= \pm 4 \pi \frac{1-\cos q R}{q^{2}}
$$

where we used the parametrization in eq. (5). Applying now the inverse transformation on eq. (25), results in

$$
\begin{aligned}
& \pm \frac{1}{(2 \pi)^{3}} \int_{0}^{\infty} \mathrm{d} q q^{2} \int_{0}^{2 \pi} \mathrm{d} \varphi \int_{0}^{\pi} \mathrm{d} \theta \sin \theta \frac{4 \pi(1-\cos q R)}{q^{2}} \sqrt{R^{2}-r^{2}} e^{-i \vec{q} \cdot \vec{r}} \\
= & \pm \frac{2}{\pi} \frac{\sqrt{R^{2}-r^{2}}}{r} \begin{cases}\frac{\pi}{2}, & r<R, \\
\frac{\pi}{4}, & r=R, \\
0, & r>R,\end{cases}
\end{aligned}
$$

which proofs the invertability. We here for concreteness pick up the Northern hemisphere potential and cast it in the more compact form,

$$
\Pi(|\mathbf{q}|)=c \frac{2 \sin ^{2} \frac{|\mathbf{q}|}{2 \hbar \sqrt{\kappa}}}{\left(\frac{|\mathbf{q}|}{\hbar \sqrt{\kappa}}\right)^{2}}, \quad c=\frac{4 G \mu}{\hbar^{2} \kappa} .
$$

It is increasing in the infrared, finite at origin, and approaches asymptotically the Coulomb propagator in the ultraviolet. In the notations of eq. (23) our result takes the form

$$
\frac{G\left(\mathbf{q}^{2}\right)}{\mathbf{q}^{2}}=c \frac{2 \sin ^{2} \frac{\mathbf{q}}{2}}{(\mathbf{q})^{2}}
$$


for a dimensionless q measured in units of $\hbar \sqrt{\kappa}$. Stated differently,

$$
G\left(\mathbf{q}^{2}\right)=2 c \sin ^{2} \frac{|\mathbf{q}|}{2}=c(1-\cos |\mathbf{q}|)=c\left(\frac{\mathbf{q}^{2}}{2 !}-\frac{\mathbf{q}^{4}}{4 !}+\frac{\mathbf{q}^{6}}{6 !}-\ldots\right),
$$

has been found. Therefore, quark physics in $\mathbf{R}^{1} \otimes S^{3}$ also predicts a finite gluon dressing function in the infrared which approaches zero in the ultraviolet. Such a type of behavior has been observed, for example, in the description of confinement phenomena [32].

\section{Conclusions}

We examined consequences of conformal symmetry in gravity-gauge duality on spectroscopic data on the lightest baryons, the nucleon and the $\Delta(1232)$. The $\mathrm{AdS}_{5} / \mathrm{CFT}_{4}$ concept has been implemented by a quark-diquark model on the $\mathrm{AdS}_{5}$ cone, conformally compactified to $\mathbf{R}^{1} \otimes S^{3}$. The description of the $q-(q q)$ system on this compact manifold has been executed in terms of the scalar conformal equation there, gauged by the field of a D3 brane whose transverse dimensions have been conformally wrapped over $S^{3}$. The spectrum of such a two-body system falls as a whole into a $\infty \mathrm{d}$ unitary representation of the conformal group, whose levels are irreps of $S O(4)$, the maximal compact group of $S O(2,4)$. For such $S O(2,4)$ irreps, the notion of "conformal bands" has been used. We observed that all nucleon and $\Delta$ resonances listed so far by the Particle Data Group distribute each fairly well over the first five levels of a respective conformal band. We did not exclude none of the states from the analyzes. We predicted a total of 32 "missing" resonances needed for the completeness of the conformal nucleon and $\Delta$ bands. Their presence or absence from the spectra relates to the degree of conformal symmetry realization in the light flavor sector. Our next result is that the gauge interaction modifies the $S^{3}$ centrifugal barrier and removes the hydrogen like degeneracies in the levels of the conformal band. This contrasts the case of the linear in the energy stationary Schrödinger equation, which keeps respecting in the interacting case the degeneracies of the $\mathcal{K}^{2}$ eigenvalue problem [22]. We furthermore observed that with the increase of the excitation energies, when the influence of the gauge potential gradually fades away, and the spectrum approaches that of the free conformal rigid rotor, the data fit becomes better. Above observations clearly point toward relevance of conformal symmetry for the spectra of the light flavor baryons. Conformal symmetry in the $N$ and $\Delta$ spectra is not an exact symmetry. The model presented accounts for this circumstance through the modification of the centrifugal barrier of the conformally invariant free geodesic motion on $S^{3}$ through the gauge interaction. This modification is responsible for the systematic $P_{2 I, 1}-S_{2 I, 1}$ mass splitting, which finds a satisfactory explanation within the framework under discussion. Encouraging, the reasonable shape of the instantaneous effective gluon propagator obtained as a Fourier transform of the gauge potential.

Work supported by CONACyT-México under grant number CB-2006-01/61286. Joint contribution based on talks by both of us at the XII Mexican Workshop on Particles and Fields.

\section{References}

[1] H. Nicolai, E. Sezgin, Y. Tanii, Nucl. Phys. B 305, 483-496 (1988).

[2] C. W. Gibbons, P. K. Townsend, Phys. Rev. Lett. 71, 3754-3757 (1993).

[3] M. Lüscher, G. Mack, Commun. Math. Phys. 41, 203-234 (1975).

[4] S. Ferrara, Ch. Fronsdal, Class. Quant. Grav. 15, 2153-2164 (1998).

[5] J. Maldacena, Phys. Rev. Lett. 80, 4859-4862 (1998).

[6] Nathan Seiberg, http://www.sns.ias.edu/ seiberg/talks.htm 
[7] Guy F. de Téramond, Stanley J. Brodsky, Phys. Rev. Lett. 94, 201601 (2005);

Stanley J. Brodsky, Guy F. de Téramond, Phys. Rev. Lett. 96, 201601 (2006).

[8] E. Klempt, Eur. Phys. J. A 38, 187-194 (2008).

[9] Jaemo Park, Piljin Yi, JHEP, 0806:011 (2008).

[10] K. Nawa, H. Suganama, T. Kojo, Phys. Rev. D 79, 026005 (2009).

[11] Gary T. Horowitz, Hirosi Ooguri, Phys. Rev. Lett. 80, 4116-4118 (1998).

[12] G. W. Gibbons, A. R. Steit, Phys. Lett. B 346, 255-261 (1995).

[13] J. L. F. Barbon, C. A. Fuertes, JHEP 0809:030 (2008).

[14] Y. S. Kim, M. E. Noz, Theory and application of the Poincaré group (D. Reidel, Dordrecht, 1986).

[15] Brian G. Wybourne, Classical groups for physicists (Wiley-Inter-science, N.Y., 1974).

[16] C. B. Compean, M. Kirchbach, Eur. Phys. J. A 33, 1-4 (2007).

[17] Gary T. Horowitz, Phys. Rev. D 50, 5204 (1994).

[18] E. Eichten, H. Gottfried, T. Kinoshita, K. D. Lane, T.M. Yan, Phys. Rev. D 17, 3090-3117 (1978).

[19] T. T. Takahashi, H. Suganuma, Y. Nemoto, H. Matsufuru, Phys. Rev. D 65, 114509-1-19 (2002).

[20] Wen-Yu Wen, Int. J. Mod. Phys. 123, 4533-4543 (2008).

[21] N. Brambilla, A. Vairo, Th. Rosch, Phys.Rev.D 72, 034021 (2005); N. Brambilla, AIP Conf.Proc.756,366-368 (2005).

[22] A. Raposo, H.-J. Weber, D. E. Alvarez-Castillo, M. Kirchbach, C. Eur. J. Phys. 5, 253-284 (2007).

[23] C. B. Compean, M. Kirchbach, J. Phys. A:Math.Theor. 42, 365301 (2009).

[24] V. D. Burkert, T. S. H. Lee, Int. J. Mod. Phys. E 13, 1035-1112 (2004).

[25] S. S. Afonin, Int. J. Mod. Phys. A 22, 4537-4586 (2007).

[26] S. G. Amsler et al., Phys. Lett. B 667, 1 (2008).

[27] M. Kirchbach, M. Moshinsky, Yu. F. Smirnov, Phys. Rev. D 64 , 114005-1-11 (2001).

[28] K. Nagata, A. Hosaka, Mod. Phys. Lett. A 19, 2397-2400 (2008).

[29] Jarl Nissfolk, Tobias Ekholm, Christer Elvingson, J. Chem. Phys. 119 (13) 6423-6432 (2003).

[30] P. O. Fedosenko, Ukr. J. Phys. 52, 607-617 (2007).

[31] A. C. Aguilar, D. Binosi, J. Papavassiliou, Phys. Rev. D 78, 025010 (2008).

[32] T. Mendez, A. Cucchieri, A. Maas, A. Mihara, Infrared propagators in MAG and Feynman gauge on the lattice [arXiv:0809.3741 [hep-lat]] 